\section{Case Reports in Oncology}

\title{
Expanding the Indication for Novel Theranostic 177Lu-Dotatate Peptide Receptor Radionuclide Therapy: Proof-of-Concept of PRRT in Merkel Cell Cancer
}

\author{
Pashtoon M. Kasi ${ }^{a} \quad$ Akash Sharma $^{\mathrm{b}} \quad$ Manoj K. Jain ${ }^{\mathrm{c}}$ \\ ${ }^{a}$ Assistant Professor, College of Medicine and Oncology, Mayo Clinic, \\ Jacksonville, FL, USA; ${ }^{b}$ Assistant Professor of Radiology, College of Medicine, Mayo Clinic, \\ Jacksonville, FL, USA; 'C Chair Division of Nuclear Medicine, Assistant Professor of \\ Radiology, College of Medicine, Mayo Clinic, Jacksonville, FL, USA
}

\section{Keywords}

177Lu-Dotatate - Lutetium - PRRT · Peptide receptor radionuclide therapy - Merkel cell cancer - Tumor-agnostic approval · Neuroendocrine tumors · Somatostatin

\begin{abstract}
The field of theranostics is a new nuclear medicine tool being utilized in the treatment of different types of cancers. It couples receptor-specific based imaging predicting and guiding response to receptor-specific based radionuclide therapies. For example, somatostatin-receptor based imaging (Gallium; 68Ga-dotatate scan) is now predicting and guiding the use of treatment with the somatostatin-receptor radiolabeled somatostatin analog (peptide receptor radionuclide therapy PRRT - Lutetium; 177Lu-Dotatate) for neuroendocrine tumors that express the somatostatin receptors. The United States Food and Drug Administration approved the use of 177Lu-Dotatate PRRT for somatostatin-receptor-positive gastroenteropancreatic neuroendocrine tumors only. Here we show proof of concept and results of an outstanding response to this novel therapy in conjunction with immunotherapy in a refractory cancer type where it has not been approved (Merkel Cell Cancer). Our results and data provide proof of principle for considering the use of this novel therapy in a tumor-agnostic approach; similar to
\end{abstract}


approval of immunotherapy for mismatch repair deficient tumors. The response demonstrated has also been unprecedented, likely secondary to use of PRRT with immunotherapy. These observations have profound and broad implications on how to move this novel field of theranostics forward for treatment of many cancer-types.

\section{Background/Case Presentation}

The field of theranostics is a new nuclear medicine tool being utilized in the treatment of different types of cancers. It couples receptor-specific based imaging predicting and guiding response to receptor-specific based radionuclide therapies. For example, somatostatin-receptor based imaging (Gallium; 68Ga-dotatate scan) is now predicting and guiding the use of treatment with the somatostatin-receptor radiolabeled somatostatin analog (peptide receptor radionuclide therapy PRRT - Lutetium; 177Lu-Dotatate) for neuroendocrine tumors that express the somatostatin receptors (Fig. 1). The United States Food and Drug Administration approved the use of $177 \mathrm{Lu}$-Dotatate PRRT for somatostatin-receptor-positive gastroenteropancreatic neuroendocrine tumors only. Here we show proof of concept and results of an outstanding response to this novel therapy in conjunction with immunotherapy in a refractory cancer type where it has not been approved. Our results and data provide proof of principle for considering the use of this novel therapy in a tumor-agnostic approach; similar to approval of immunotherapy for mismatch repair deficient tumors. The response demonstrated has also been unprecedented, likely secondary to use of PRRT with immunotherapy. These observations have profound and broad implications on how to move this novel field of theranostics forward for treatment of many cancer-types.

Earlier on January 26, 2018, based on a landmark phase-3 clinical trial, the United States Food and Drug Administration approved the use of 177Lu-Dotatate PRRT for somatostatinreceptor-positive gastroenteropancreatic neuroendocrine tumors (NETs) [1]. The somatostatin-receptor-positivity is determined by using the newly evolving field of theranostics using radioisotopes or radionuclides to label peptides of interest. In this particular case, the somatostatin-receptor positivity is determined by the well-known octreotide scan (octreascan) or the more recent Gallium (68Ga)-dotatate scan. Figure 1 simplistically illustrates the theranostics of 177Lu-Dotatate PRRT for somatostatin-receptor-positive gastroenteropancreatic neuroendocrine tumors.

However, beyond neuroendocrine tumors of the gastrointestinal tract, other tumors can exhibit this somatostatin-receptor-positivity. These include lung NETs, pheochromocytomas, thymic tumors and prostate cancer. Since this is a predictive marker of response, this should make PRRT a valid radiotherapeutic option for these tumors beyond just NETs.

We present a case of a refractory Merkel cell cancer that within days of one off-label dose of 177Lu-Dotatate PRRT showed dramatic reduction in the size and symptoms from all palpable tumors (Fig. 2). A single-photon emission computerized tomography (SPECT) scan done post therapy shows the 177Lu-Dotatate therapy distributing itself in all the locations of 68Gapositivity done prior to this treatment (Fig. 2B). The follow-up 68Ga-scan within a month shows near complete resolution of all the metastases with some left over avidity but no palpable/visible tumors. 


\section{Discussion}

Our report has several novel observations and raises a key question that has implications for patients with multiple types of cancers. Firstly, this provides proof of concept for considering the use of this novel therapy in a tumor agnostic approach; similar to approval of immunotherapy for mismatch repair deficient tumors [2]. Theranostics is receptor-specific, not tumor-specific. If a tumor expression can be shown either histologically or radiographically here by the use of 68Ga-scan, receptor based treatments like 177Lu-PRRT should then work for them.

Secondly, we report on dramatic early and rapid response in a novel tumor type (Merkel cell) in a refractory setting $[3,4]$. This response is unprecedented as well. In the original phase3 clinical trial for patients with NETs, there was only 1 patient with a complete response. The remainder primarily had disease control.

Thirdly, this patient had been on anti-protein programmed death-ligand 1 (PD-L1) immunotherapy (avelumab) to which the tumor was refractory. We reinitiated its use pre- and postPRRT while we worked on how to get the PRRT for this patient under compassionate use. The rationale behind this was secondary to the proposed synergy given the synergy between radiation therapies and immunotherapy [5]. This is also one of the first reports showing its feasibility and efficacy with PRRT.

The current National Comprehensive Cancer Network (NCCN) guidelines have included the use of PRRT for lung NETs, thymic tumors, pheochromocytomas, paragangliomas and NETs of unknown primary as well, even though there are no randomized controlled trials supporting its use. The field of theranostics where somatostatin-receptor based imaging is guiding the use of somatostatin-receptor radiolabeled somatostatin analog should, therefore, not be limited to specific tumor types. A tumor agnostic trial utilizing PRRT for patients with cancers that are somatostatin receptor positive with or without immunotherapy would be a novel strategy to expand the indication of this novel therapy. Finally, it would not be unreasonable to expand the current indication of this novel therapy for patients whose tumors exhibit somatostatin-receptor positivity.

In parallel, the costs of some of these treatments need to be revisited. One dose of PRRT is more than USD 100,000 factoring in all the costs of delivery of the radionuclide. This is exclusive of costs of novel drugs like immunotherapy, the costs of which are already a focus of many ongoing debates. In this particular instance, the PRRT costs given the circumstances had to be done out-of-pocket, which is obviously not a sustainable strategy. Later, insurance covered it after appeals. While dramatic and unprecedented, the cost of this and any other novel therapies for many cancers alike is an area of ongoing concern.

\section{Acknowledgements}

The authors are deeply indebted to the patient and his family for allowing us to present the anonymous information as noted. Thanks are also due to Mayo Media Services for the illustration (Fig. 1).

\section{Statement of Ethics}

The authors have no ethical conflicts to disclose. 


\section{Disclosure Statement}

The authors declare no competing interests pertaining to this article. Author PMK has participated in advisory board for Taiho (2017) and Ipsen (2018) (to institution).

\section{Author Contributions}

Author PK wrote the initial draft, which was then edited with contributions from authors MJ and AS. Author AS provided the images of 177Lu-therapy and pre- and post-68Ga-scan. PK reformatted all this. All authors for publication approved the final draft.

\section{References}

1 Strosberg J, El-Haddad G, Wolin E, Hendifar A, Yao J, Chasen B, et al.; NETTER-1 Trial Investigators. Phase 3 Trial of 177Lu-Dotatate for Midgut Neuroendocrine Tumors. N Engl J Med. 2017 Jan;376(2):125-35.

2 Le DT, Durham JN, Smith KN, Wang H, Bartlett BR, Aulakh LK, et al. Mismatch repair deficiency predicts response of solid tumors to PD-1 blockade. Science. 2017 Jul;357(6349):409-13.

3 Schmidt MC, Uhrhan K, Markiefka B, Hasselbring L, Schlaak M, Cremer B, et al. (68)Ga-DotaTATE PET-CT followed by Peptide Receptor Radiotherapy in combination with capecitabine in two patients with Merkel Cell Carcinoma. Int J Clin Exp Med. 2012;5(4):363-6.

4 Basu S, Ranade R. Favorable Response of Metastatic Merkel Cell Carcinoma to Targeted 177Lu-DOTATATE Therapy: Will PRRT Evolve to Become an Important Approach in Receptor-Positive Cases? J Nucl Med Technol. 2016 Jun;44(2):85-7.

5 Weichselbaum RR, Liang H, Deng L, Fu YX. Radiotherapy and immunotherapy: a beneficial liaison? Nat Rev Clin Oncol. 2017 Jun;14(6):365-79. 


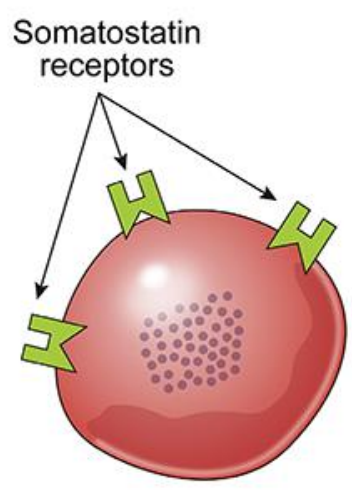

Cancer cell

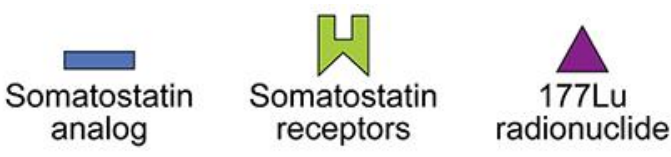

Somatostatin analog (peptide) linked to $177 \mathrm{Lu}$ radionuclide

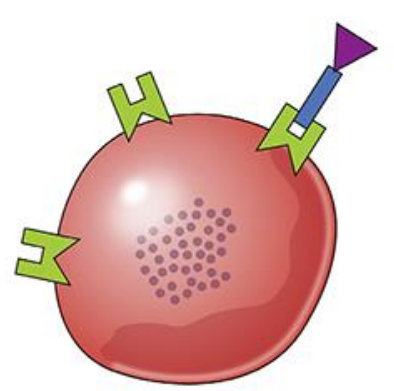

177Lu-somatostatin analog complex attaching to somatostatin receptors on cell surface

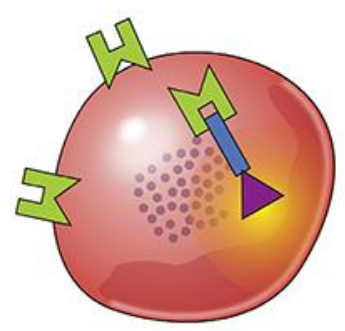

177Lu-somatostatin analog complex internalizing into the cell and emitting therapeutic beta radiation

Fig. 1. Simplistic overview illustrating theranostics of recently FDA approved radionuclide therapy (177Lu-Dotatate peptide receptor radionuclide therapy - PRRT) for somatostatin-receptor-positive neuroendocrine tumors. Illustration: (Steven D. Orwoll - Mayo Clinic Media; adaptation from Kasi PM et al.: A Care Process Model to Deliver 177Lu-Dotatate Peptide Receptor Radionuclide Therapy for Patients with Neuroendocrine Tumors. Front. Oncol. | doi: 10.3389/fonc.2018.00663). 


\section{Case Reports in Oncology}

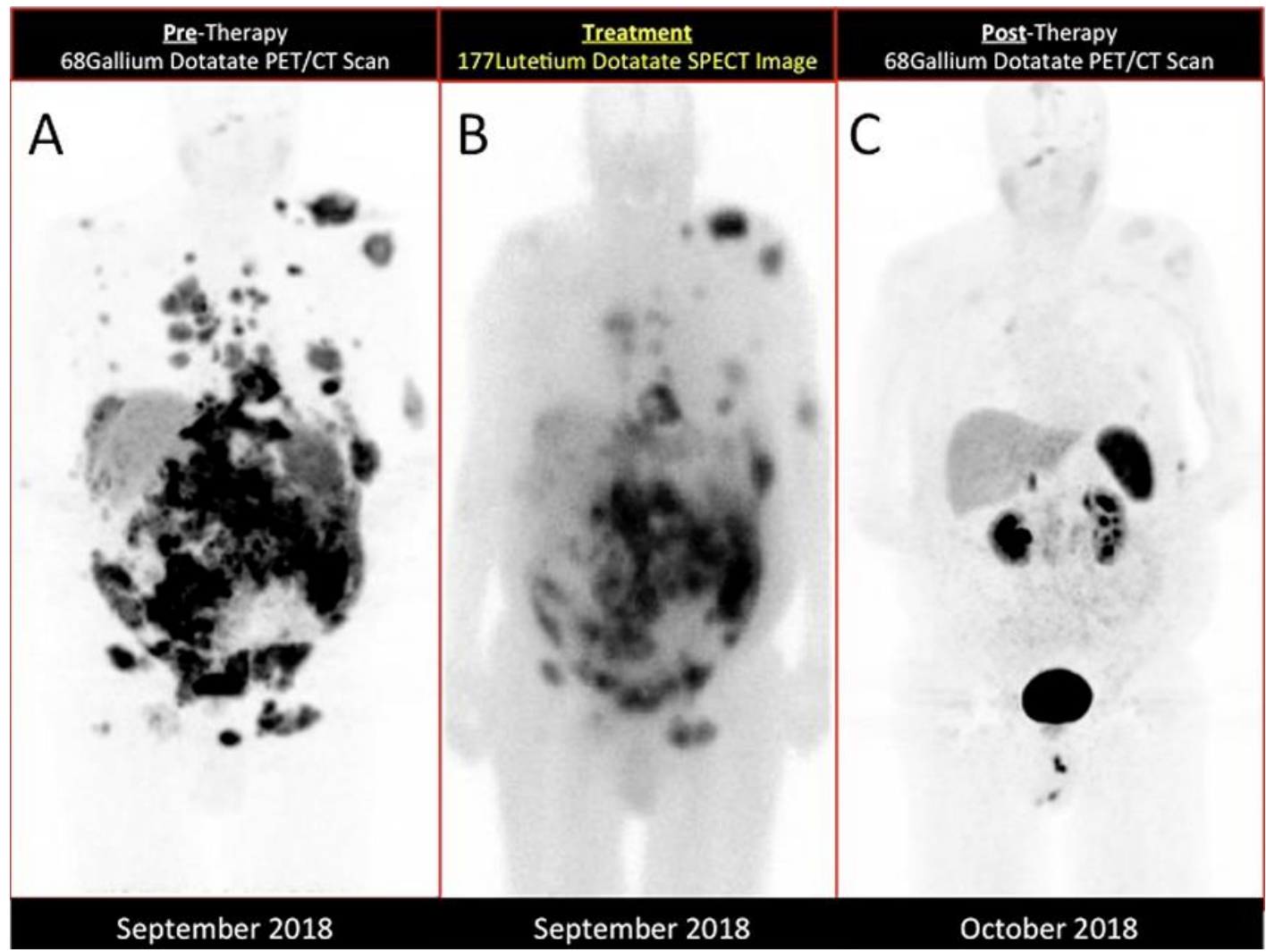

Fig. 2. Panel on the left (A) shows the diagnostic images (pre-treatment) from 68Ga dotatate PET/CT scan done prior to treatment showing immense somatostatin receptor positivity in a patient with Merkel Cell Cancer. The cancer metastases can be seen in many parts of the body including carcinomatosis (spread to the abdomen), bones including sternum/ribs and large metastases palpable under the skin (reflecting high disease burden). Panel in the center (B) provides proof of principle showing the radiolabeled somatostatin analog (177Lu-Dotatate) therapy distributing itself in all the locations of somatostatin-receptor positivity. Panel on the right (C) shows the diagnostic images (post-treatment) from 68Ga dotatate PET/CT scan done 1-month post-treatment showing near complete resolution of all the metastases. Note: The SPECT images (B) are acquired about four hours after the PRRT infusion and due to differences in technique appear different as compared to the pre-treatment PET scan (A). The spleen, liver, kidneys and bladder/urinary tract light up normally on the 68Ga-scan. 